
package for the study of particle dynamics modeling in the atmospheric boundary layer and in the model wind farm. The Euler-Lagrangian approach is used to describe particle dynamics. Two new solvers based on ABLSolver and pisoTurbineFoam.ALM have been developed to simulate particle dynamics. The calculation results for the case of a neutral boundary layer and a model wind farm with 14 model wind turbines are presented. Graphs for the distribution of particles with different diameters in height are given Between 72 and 96 cores were used to calculate one example.

Keywords: SOWFA, library, flow, simulation, solver, particles, atmospheric boundary layer, wind farm, velocity, viscosity, turbulence, altitude

планетарном пограничном слое и в модельном ветропарке

К.Б. Кошелев, ORCID: 0000-0002-7124-3945<koshelevkb@mail.ru> C.B. Стрижак, ORCID:0000-0001-5525-5180<s.strijhak@ispras.ru> Институт системного программирования им. В.П. Иванникова РАН, 109004, Россия, г. Москва, ул. А. Солженищына, д. 25

Аннотация. В настоящее время в РФ активно ведется строительство новых ветропарков. Вопросы изучения физических процессов являются актуальными, так как действующие ветропарки оказывают влияние на микроклимат и экологию. В ветропарках возможно появление и движение жидких и твердых частиц. При исследовании двухфазных потоков, содержащих взвесь аэрозольных частиц (дисперсная фаза) в несущей среде (дисперсионная среда) в атмосфере важно правильно выбирать основные параметры, определяющие систему, и адекватно описать реальный процесс при помощи сформулированной математической модели. Работа посвящена разработке новых решателей на базе библиотеки SOWFA в составе открытого пакета OpenFOAM 2.4.0 для изучения моделирования динамики частищ в планетарном (атмосферном) пограничном слое и в модельн ретре б овых реш. . ретропарка с 14 модельными ветроустановками. Приведены графики для распределения частиц с вычислительных ядер.

Ключевые слова: SOWFA; библиотека; течение; моделирование; решатель; частицы; атмосферный пограничный слой; ветропарк; скорость; вязкость; турбулентность; высота

Для цитирования: Кошелев К.Б., Стрижак С.В. Моделирование динамики частиц в планетарном пограничном слое и в модельном ветропарке. Труды ИСП РАН, том 31, вып. 6, 2019 г., стр. $177-$ 186. DOI: $10.15514 /$ ISPRAS-2019-31(6)-10

Благодарности: Работа выполнена при финансовой поддержке РФФИ (грант № 17-07-01391).

\section{Simulation of particle dynamics in planetary boundary layer and in a model wind farm}

K.B. Koshelev, ORCID: 0000-0002-7124-3945<koshelevkb@mail.ru> S.V. Strijhak, ORCID:0000-0001-5525-5180<s.strijhak@ispras.ru> Ivannikov Institute for System Programming of the Russian Academy of Sciences, 25, Alexander Solzhenitsyn st., Moscow, 109004, Russia

Abstract. Currently, Russia is actively building new wind farms. The issues of studying physical processes are relevant, as the existing wind farms have an impact on the microclimate and ecology. In wind farms, the appearance and novers containing a suspension of aerosol particles (dispersed phase) in the carrier medium (dispersion medium) adequately describe the real process using the formulated mathematical model. The work is devoted to the
For citation: Koshelev K.B., Sttrijhak S.V. Simulation of particle dynamics in planetary boundary laye and in a model wind farm. Trudy ISP RAN/Proc. ISP RAS, vol. 31, issue 6, 2019. pp. 177-186 (in Russian). DOI: 10.15514/ISPRAS-2019-31(6)-10

Acknowledgements. This work was supported by the Russian Foundation for Basic Research (Grant No 17-07-01391).

\section{1. Введение}

В настоящее время в РФ активно ведется строительство новых ветропарков. Вопросы изучения физических процессов являются актуальными, так как действующие ветропарки оказывают влияние на микроклимат и экологию.

Изучение движения вихревых структур в атмосферном пограничном слое (АПС) с учетом влияния стратификации среды, орографии местности, вращения Земли, изменения тепловых потоков и градиента давления важно с точки зрения задач моделирования климата, изучения физических процессов при проектировании ветропарков и оценки их эффективности работы на территории РФ [1,2]. Для моделирования процессов в атмосферном пограничном слое характерно использование вихреразрешающего моделирования с использованием различных моделей для подсеточной турбулентной вязкости. Известно, что для работы ветропарков характерно условия существования нейтральной и устойчивой стратификации. Полученные результаты расчета для АПС могут быть использованы в качестве начальных данных для моделирования параметров ветропарка.

В ветропарках возможно появление и движение жидких и твердых частиц. При исследовании двухфазных потоков, содержащих взвесь аэрозольных частиц (дисперсная фаза) в несущей среде (дисперсионная среда) в атмосфере важно правильно выбирать основные параметры, определяющие систему, и адекватно описать реальный процесс при помощи сформулированной математической модели. Дисперсионной средой может быть жидкость (Ж) или газ (Г), дисперсной фазой могут быть твердые частицы $(\mathrm{T})$, капли жидкости (Ж) или газовые пузырьки (Г) [3-5]

Различные частицы, переносимые воздухом в атмосферном пограничном слое, могут быть причиной образования наростов и повреждений на горизонтальных ветроэлектрических установках (ВЭУ) в действуюших ветропарках. Такие частищы как капли дождя, песчинки пыли, ледяные снежинки, градины, различные насекомые являются главными причинами загрязнения поверхности лопасти ВЭУ и снижения производительности ВЭУ во время эксплуатации [6-8].

В 2019 году на территории Республика Адыгея и в Ставропольском крае ведется строительство двух новых ветропарков. Данные регионы расположены в южных широтах, поэтому в летнее время возможно появление различных насекомых (стрекозы, жуки, бабочки, плодовые мошки и другие). Как показывает исследование, проведенное в немецком центре DLR, максимальная плотность насекомых составляет 9.0 кг/км3 на высотах 0-400 метров [9]. Скорость движения плодовых мошек может достигать 12-14 метров. В дневное время конвективные шлейфы с вертикальной скоростью ветра (плюмы) 

пограничного слоя. Радиолокационный анализ выявил до тысячи раз более высокую концентрацию насекомых в таких шлейфах по сравнению с окружающей средой. Большинство мелких, пассивно летающих насекомых остается в пределах таких шлейфов и добирается высот в 1000 метров или более над уровнем Земли, прежде чем они начинают перемещаться с ветром в горизонтальном направлении.

Более крупные насекомые, особенно когда они движутся ночью без влияния конвективных шлейфов, могут выбирать нижние слои для своей миграции. Для экономии энергии и поддержания сбалансированной температуры тела во время полета они могут выбрать слой с максимальной скоростью ветра и адекватной температурой воздуха Общеизвестно, что насекомые обычно избегают летать, когда есть сильный ветер и дождь, так как при высокой турбулентности вблизи Земли полет и навигация затруднены. В этом случае насекомые предпочитают прятаться и оставаться внизу. Миграция начинается в спокойные погодные условия, когда скорость ветра на уровне Земли достаточно низкая. Однако, как только насекомые достигают поверхностного слоя, скорость перемещения может варьироваться от 20 до 100 км/ч $(5-25$ м/с). К сожалению, такое поведение приводит их непосредственно в критический диапазон высот работы роторов ветроэлектрических установок (ВЭУ)

Пространственная поверхность лопасти ВЭУ может аккумулировать грязь вокруг передней кромки. Более того, соударение частиц, резкие скачки температуры, циклы замораживания-оттаивания могут вызывать образование трещин в покрытии, приводящие к эрозии поверхности, расслоению ядра материала и коррозионному разрушению внутренней структуры композиционного материала. Гладкая первоначальная поверхность лопасти может значительно измениться по сравнению с начальным состоянием, и образовавшаяся шероховатость может значительно снизить величину вырабатываемой мощности ВЭУ.

На поверхности лопасти в дозвуковом пограничном слое могут происходить существенные изменения и образовываться локальный отрыв потока, если толщина “нароста" сравнима с толщиной пограничного слоя. Коэффициент трения существенно увеличивается в связи с ламинарно-турбулентным переход в пограничном слое. С аэродинамической точки зрения снижение аэродинамической эффективности ВЭУ связано с коэффициентом сопротивления как результат взаимодействия поверхности лопасти с окружающимися частицами. Другая важная проблема связана с увеличением уровня шума, измеряемого в Дб. Изменение структуры поверхности может повысить уровень шума в пограничном слое.

В связи с этим операторы ветропарков вынуждены проводить проверку и обслуживание лопастей ВЭУ. С технологической точки зрения компаниями разработаны специальные покрытия на основе полиэритана.

Изучение движения частиц в ветропарке также важно с точки зрения оценки дефицита скорости для ВЭУ, расположенных в разных рядах, и оценки эффекта инжекции воздушных масс [10].

Поэтому изучение динамики частиц в ветропарках и их влияния на поверхность лопастей ВЭУ с помощью математического моделирования является актуальной задачей. Нами было выбрано программное обеспечение, в основе которого используется открытая библиотека SOWFA для моделирования физических процессов в АПС и расчета параметров турбулентного течения в ветропарках $[11,12]$.

\section{2. Программное обеспечение}

Расчёт описанной задачи произведём в пакете OpenFOAM версии 2.4.0. Используется библиотека SOWFA, предназначенная для решения моделирования течений в

атмосферном пограничном слое и в ветропарке. Данная библиотека была разработана в 2010 году в лаборатории NREL. Авторы данной статьи приняли решение продолжить разработку новых решателей в библиотеке SOWFA в OpenFOAM версии 2.4.0. Если бы использовалась последняя версии OpenFOAM 7.0, то потребовалось бы переписать значительную часть исходного кода библиотеки SOWFA. Численный метод в данной библиотеке основан на методе конечных объёмов. Математическая модель включает в себя законы сохранения массы, количества движения и энергии.

B составе библиотеки SOWFA имеется несколько проблемно-ориентированных решателей, в том числе для расчёта атмосферного пограничного слоя (решатель ABLSolver) и физических параметров в ветропарке (решатель windPlantSolver) с использованием метода крупных вихрей и динамической лагранжевой модели Смагоринского [13,14]. В составе библиотеки имеется несколько моделей для подсеточной турбулентной вязкости и специальные граничные условия для задания скорости и температуры. Модель плоских сечений профиля (Actuator Line Model - ALM) может быть использована для расчёта течения вблизи вращающихся лопастных турбин на фиксированной расчётной сетке, что значительно экономит вычислительные ресурсы и упрощает процесс счёта. Параллельные вычисления могут выполняться с использованием стандартного метода декомпозиции расчётной области.

\section{3. Математическая модель}

Решатель ABLSolver предназначен для моделирования атмосферного пограничного слоя (АПС) в случае плоской поверхности земли при наличии температурного градиента (стабильный, нестабильный, нейтральный АПС). Математическая модель включает в себя уравнения (1) - (8)

$$
\begin{gathered}
\frac{\partial \bar{u}_{j}}{\partial x_{j}}=0 \\
\frac{\partial \bar{u}_{i}}{\partial t}=-\frac{\partial}{\partial x_{j}}\left(\bar{u}_{j} \bar{u}_{i}\right)-\frac{\partial R_{i j}^{D}}{\partial x_{j}}-\frac{\partial \tilde{p}}{\partial x_{i}}-\left(\frac{\partial \tilde{p}}{\partial x_{i}}\right)^{d}+\left(1-\frac{\bar{\theta}}{\bar{\theta}^{0}}\right) g_{i}+\epsilon_{i j} f^{c} \bar{u}_{j} \\
\frac{\partial \bar{\theta}}{\partial t}=-\frac{\partial}{\partial x_{j}}\left(\bar{u}_{j} \bar{\theta}\right)-\frac{\partial R_{\theta j}}{\partial x_{j}} \\
R_{i j}^{D}=-2 v^{S G S} \bar{S}_{i j} \\
\bar{S}_{i j}=\frac{1}{2}\left(\frac{\partial \bar{u}_{i}}{\partial x_{j}}+\frac{\partial \bar{u}_{j}}{\partial x_{i}}\right) \\
v^{S G S}=\left(C_{S} \Delta\right)^{2}\left(2 \bar{S}_{i j} \bar{S}_{i j}\right)^{\frac{1}{2}} \\
R_{\theta j}=-\frac{v^{S G S}}{P r_{t}} \frac{\partial \bar{\theta}}{\partial x_{j}} \\
\frac{\partial R_{i j}^{D}}{\partial x_{j}}=-\frac{\partial}{\partial x_{j}}\left(v^{S G S} \frac{\partial \bar{u}_{i}}{\partial x_{j}}\right)-\frac{\partial}{\partial x_{j}}\left[v^{S G S}\left(\frac{\partial \bar{u}_{j}}{\partial x_{i}}-\frac{2}{3} \frac{\partial \bar{u}_{k}}{\partial x_{k}} \delta_{i j}\right)\right]
\end{gathered}
$$

где $\bar{u}_{j}$ - скорость, $\tilde{p}$ - давление, $R_{i j}^{D}=R_{i j}-R_{k k} \delta / 3-$ тензор напряжения, $\left(\frac{\partial \tilde{p}}{\partial x_{i}}\right)^{d}-$ градиент давления, $\bar{\theta}$ - потенциальная температура, $f^{c}$ - параметр Кориолиса.

Для описания динамики частиц используется эйлер-лагранжев подход. Модификация решателя ABLSolver заключалась в добавлении модели кинематического облака частиц [15]. В данной реализации решателя были добавлены слагаемые, учитывающих движение частиц и их взаимодействие с воздушным потоком. Тепловое взаимодействие пока не учитывалось. 
Фактически решатель ABLSolverP был разработан путем добавления к решателю ABLSolver следующих строк (выделены жирным шрифтом):

Модуль UEqn.

// Solve the momentum equation

\#include "computeCoriolisForce.H"

\#include "computeBuoyancyTerm.H"

fvVectorMatrixuEqn

(

$\mathrm{fvm}:$ : ddt (U)

// time derivative

U) // convection

(U)

frve: div(Rwali)

fCoriolis

prhol * kinematicCloud.sU(U)

Coriolis force

// mesoscale source terms )

// momentum form parti

UEqn. relax () ;

Также были внесены ссылки на необходимые модули в файлы проекта.

Решатель pisoTurbineFoam.ALM предназначен для моделирования течения в ветропарке с использованием метода ActuatorLineModel. Его модификация также заключалась в добавлении кинематического облака частиц.

Дополнительно был разработан новый решатель partpisoTurbineFoam.ALM для моделирования облака частиц в ветропарке. Решатель partpisoTurbineFoam.ALM был разработан путем добавления к решателю pisoTurbineFoam.ALM следующих строк (выделены жирным шрифтом):

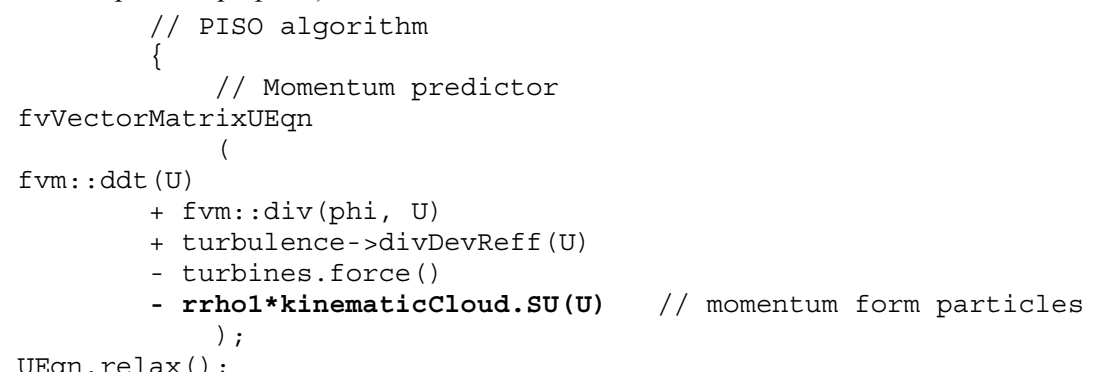

\section{4. Объекты исследования}

\section{1 Атмосферный пограничный слой}

Известно, что для атмосферного пограничного слоя характерно наличие крупных вихревых структур, которые оказывают влияние на развитие турбулентности у поверхности [16-18]. Для изучения физических процессов с помощью вихреразрешающего моделирования обычно используют прямоугольную расчетную область [18].

Задача об изучение образования кластеров аэрозолей с учетом влияния влажности, тепловых потоков в атмосферном пограничном слое с использованием данных метеонаблюдений является актуальной задачей в связи изучением микроклимата и оценки уровня солнечной радиации, достигающей поверхности Земли $[19,20]$.

Будем рассматривать следующую постановку задачи: имеется область в форме параллелепипеда с заданными размерами 3000 × 1000 х 3000 метров. Исследуются физические параметры в нейтральном пограничном слое [18, 21,22]. На входе в расчетную область задается профиль скорости и вводится облако твердых частиц.

Для задания тестового примера теперь надо иметь, по крайней мере один дополнительный файл в каталоге constant - Cloudlproperties для описания характеристик частиц. Секция interpolationSchemes должна содержать схемы для переменных rhok, U, nuSGS. В качестве частиц рассматриваются частицы песка. Типичная плотность песка равна 2550 кг/м3. В нижеприведенном примере модель ввода частиц (парселей) - модель сечения. В расчетную область вводится 100 кг частиц за 10 секунд со скоростью ввода 10 частиц (парселей) в секунду и собственной скоростью 8 м/с. Диаметр частиц задается фиксированным $2.5 \cdot 10^{-4}$ метра. Ввод частиц осуществлялся через входное сечение для скорости газа. На выходной и боковых границах задавалось циклические граничные условия. Таким образом введенные частицы не покидали расчетную область.

Шаг по времени для данной задачи в эйлерово-лагранжевой постановке пришлось уменьшить в 5 раз по сравнению с аналогичной задачей без частиц. Положение частиц (парселей) при «установившемся» режиме течения приведено на рис. 1. Оказалось, что частицы под влиянием конкурирующих сил тяжести и турбулентной диффузии занимают примерно нижнюю треть пространственной области.

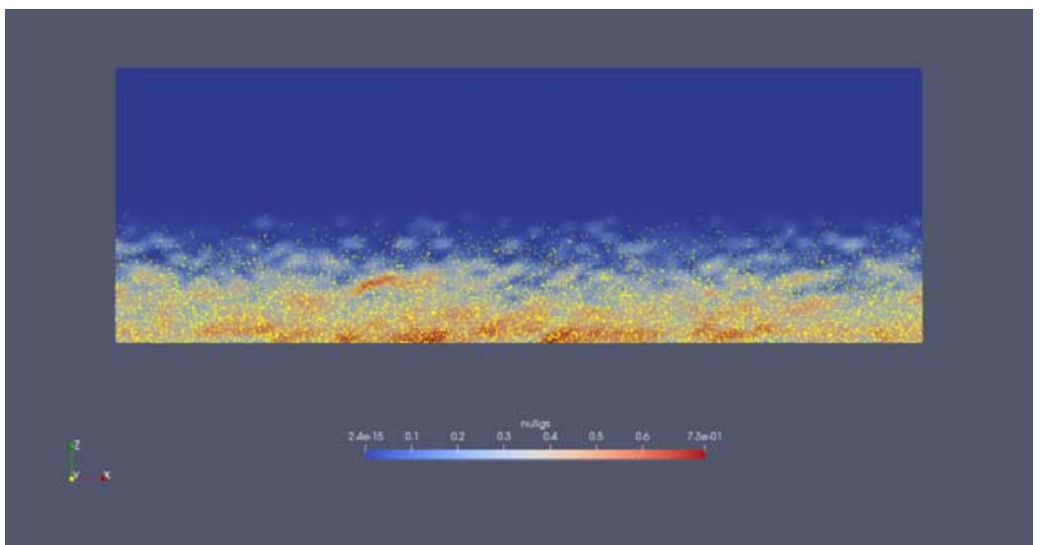

Рис.1. Положение частии при «установившемся» течении на фоне турбулентной вязкости Fig. 1. Parcel position in a "steady" flow against a background of turbulent viscosity

\section{2 Модельный Ветропарк}

Рассматривается модельный ветропарк с движением твердых частиц [23]. Пространственная область в данном примере имеет размеры 1м х 5м х 9м. В модельном ветропарке расположено 14 модельных ветроэлектрических установок (ВЭУ) Расположение имитаторов ветроустановок соответствует расположению 14 ВЭУ в ветропарке, расположенном в Ульяновской области РФ. Геометрические данные для имитаторов ВЭУ взяты из работ [21,23]. Расчет течения проводился с использованием

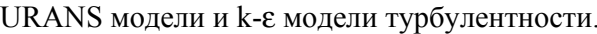

Валидация решателя без частиц рisoTurbineFoam.ALM ранее проводилась на модельных задачах с 2, 12, 14 имитаторами ВЭУ в нейтральном атмосферном пограничном слое [2428]. Было получено хорошее совпадение с данными эксперимента в климатических трубах, ошибка при вычислении значений коэффициента мощности Ср для ВЭУ составила менее $5 \%$ 

в каталоге constant - стандартный $\mathrm{g}$ и kinematicCloudlproperties. Секция interpolationSchemes должна содержать схемы для переменных rhok, U, nut (при использовании RANS k-e модели турбулентности). В нижеприведенном примере модель ввода частиц (парселей) - модель сечения. Вводится масса частиц $\mathrm{m}=0.0006$ кг со $1.5 \mathrm{M} / \mathrm{c}$. Диаметр частиц фиксированный и равен $10^{-5} \mathrm{M}$. Ввод частиц осуществлялся через входное сечение для скорости газа. На боковых границах задавалось циклические граничные условия. Процесс вычислений полагался установившимся, когда за 1 с модельного времени количество введенных и покинувших область частиц совпадало.

\section{5. Результаты расчета}

Положение частиц (парселей) при «установившемся» режиме течения приведено на рис. 1. Оказалось, что частицы вод влиянием конкурирующих сил тяжести и турбулентной диффузии занимают примерно нижнюю треть пространственной области. На рис. 2 показано положение частиц в ветропарке в момент времени $\mathrm{t}=7.4$ секунда.

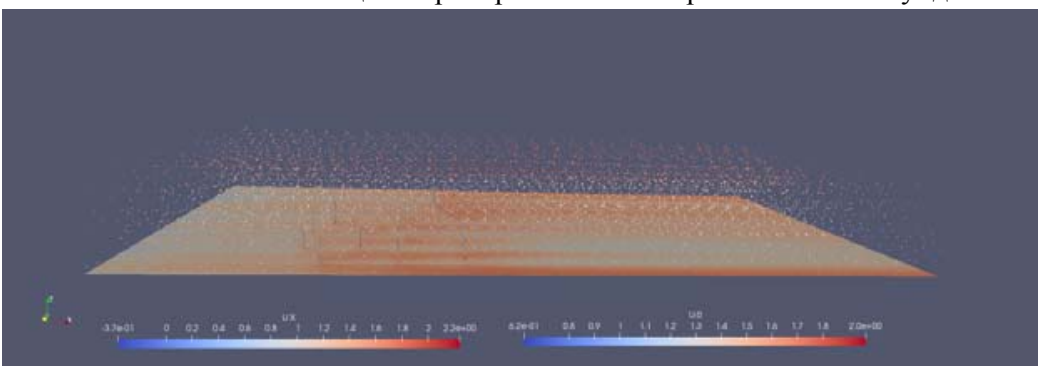

Рис. 2. Положение парселей в ветропарке в момент времени $t=7.4$ секунд Fig. 2. The position of the parcels in the wind farm at time $t=7.4$ seconds

На рис. 3 для примера с атмосферным пограничным слоем красным цветом выделено распределение частиц по высоте с диаметром 25 мкм, а синим цветом - с диаметром 50 мкм. Как видно особой разницы в распределение частиц по высоте нет, так как частиц относительно мало и на турбулентность они не влияют. Там, где заканчивается влияние турбулентности (выше примерно чуть более 400 м) все частицы под влиянием силы тяжести опускаются ниже. Впрочем, влияние силы тяжести наблюдается на всех высотах.

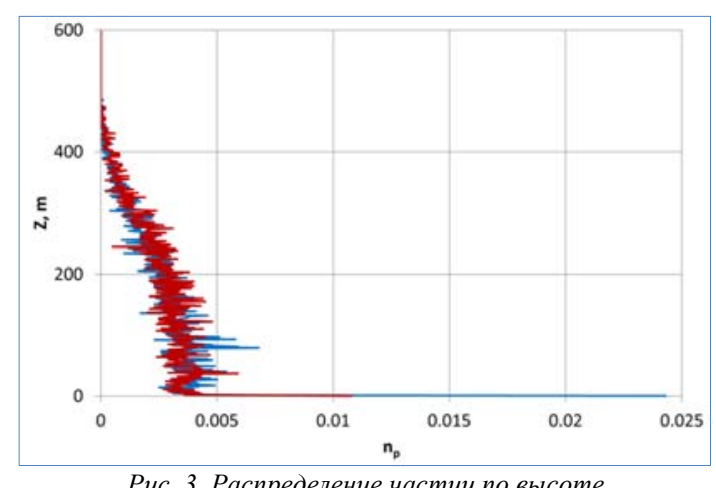

Pис. 3. Распределение частии по высоп

Fig. 3. Parcel distribution by height

На Рисунке 4 для случая модельного ветропарка [28] показано распределение частиц по высоте с диаметром 10 мкм. Как видно, картина в корне отличается от реального примера с атмосферным пограничным слоем. Влияние ВЭУ и силы тяжести распознать достаточно сложно. В дальнейшем требуется проведение дополнительных исследований для изучения данного вопроса.

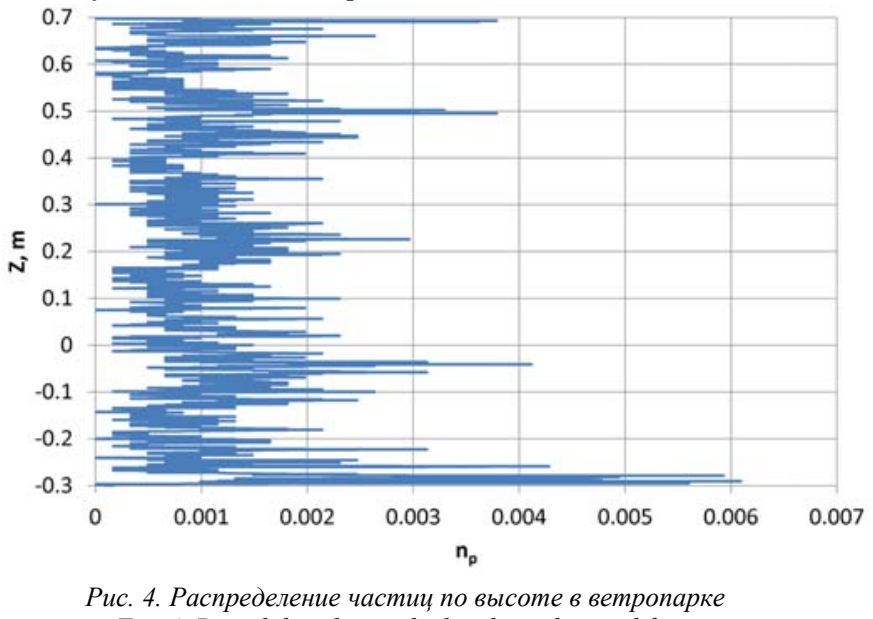

Fig. 4. Parcel distribution by height in the wind farm

\section{6. Заключение}

В статье рассмотрены возможности библиотеки SOWFA для решения прикладных задач механики сплошной среды в области ветроэнергетики. Исследование процессов турбулентного движения в атмосферном пограничном слое и в модельном ветропарке предложено осуществлять с применением средств отслеживания облака частиц. В статье приведен пример добавления модели облака частиц в состав решателя ABLSolver и pisoTurbineFoam.ALM. Разработаны два новых решателя для моделирования динамики части в составе библиотеки SOWFA. Для демонстрации работы новых решателей представлены результаты расчета поля турбулентной вязкости, для модельного ветропарка с 14 ВЭУ. Вычисления были проведены с использованием ресурсов вычислительного кластера web-лаборатории UniCFD ИСП РАН. Для расчета одного примера было использовано от 72 до 96 вычислительных ядер.

\section{Список литературы / References}

[1]. Лыкосов В.Н., Глазунов А.В., Кулямин Д.В., Мортиков Е.В., Степаненко В.М Суперкомпьютерное моделирование в физике климатической системы: учебное пособие. М., Изд.-во Московского университета, 2012 г., 408 стр. / Lykosov V.N., Glazunov A.V., Kulyamin D.V., Mortikov E.V., Stepanenko V.M. Supercomputer modeling in the physics of the climate system a training manual. M., Publishing House of Moscow University, 2012, 408 p. (in Russian).

2]. Зилитинкевич С.С. Атмосферная турбулентность и планетарные пограничные слои. М., Физматлит, 2014 г., 252 стр. / Zilitinkevich S.S. Atmospheric turbulence and planetary boundary layers. M., Fizmatlit, 2014, 252 p. (in Russian).

[3]. Алоян А.Е. Динамика и кинетика газовых примесей и аэрозолей в атмосфере. Курс лекций. М., ИВМ РАН, 2002 г., 201 стр. / Aloyan A.E. Dynamics and kinetics of gas impurities and aerosols in the atmosphere. Lecture course. M., INM RAS, 2002, 201 p. (in Russian). 
[4]. Береснев С.А., Грязин В.И. Физика атмосферных аэрозолей: Курс лекций. Екатеринбург, Издво Урал. ун-та, 2008 г., 227 стр. / Beresnev S.A., Gryazin V.I. Physics of Atmospheric Aerosols: Lecture Course. Yekaterinburg, Publishing House of Ural University, 2008, 227 p. (in Russian).

[5]. Архипов В.А., Усанина А.С. Движение аэрозольных частиц в потоке: учебное пособие. Томск, Издательский дом Томского государственного университета, 2013 г., 92 стр. / Arkhipov V.A., Usanina A.S. The movement of aerosol particles in a stream: a training manual. Tomsk, Publishing House of Tomsk State University, 2013, 92 p. (in Russian).

[6]. Fiore G., Selig M. S. A Simulation of Operational Damage for Wind Turbines. In Proc. of the 32nd AIAA Applied Aerodynamics Conference, 2014. pp. 1-19.

[7]. Fiore G., Fujiwara G.E.C., Selig M.S. A Damage Assessment for Wind Turbine Blades from Heavy Atmospheric Particles. In Proc. of the 53rd AIAA Aerospace Sciences Meeting, 2015, pp. 1-22.

[8]. Sareen A., Sapre C.A., Selig M.S. Effects of leading edge erosion on wind turbine blade performance. Wind Energy, vol. 17, issue 10, 2014, pp. 1531-1542.

[9]. Trieb F. Interference of Flying Insects and Wind Parks. Study Report. Deutsches Zentrum für Luftund Raumfahrt (DLR), 2018, $30 \mathrm{p}$

[10].Breton S-P, Sumner J., Sorensen J.N., Hansen K.S., Sarmast S., Ivanell S. A survey of modeling methods for high-fidelity wind farm simulations using large eddy simulation. Philosophical Transactions of the Royal Society A: Mathematical, Physical and Engineering Sciences, vol. 375, issue 2091, 2017, article no. A 375:20160097.

[11].Churchfield M.J., Moriarty P.J., Vijayakumar G., Brasseur J.G. Wind Energy-Related Atmospheric Boundary Layer Large-Eddy Simulation Using OpenFOAM. In Proc. of the 19th Symposium on Boundary Layers and Turbulence, 2010, $23 \mathrm{p}$.

[12].Churchfield M.J., Lee. S., Michalakes J., Moriarty P.J. A numerical study of the effects of atmospheric and wake turbulence on wind turbine dynamics. Journal of Turbulence, vol. 13, 2012, article no. 13.

[13]. Sagaut P. Large eddy simulation for incompressible flows: an introduction. Springer, Berlin, 2001 , $319 \mathrm{p}$.

14]. Meneveau C, Lund T S, Cabot W H. A Lagrangian dynamic subgrid-scale model of turbulence. Journal of Fluid Mechanics, vol. 319, 1996, pp. 353-385.

[15].Крапошин M.B., Стрижак С.В. Проблемно-ориентированная библиотека SOWFA для решения прикладных задач ветроэнергетики. Труды ИСП РАН, том 30, вып. 6, 2018 г., стр. 259-274 M.V. Kraposhin, S.V. Strijhak The problem-oriented library SOWFA for solving the applied tasks of wind energy. Trudy ISP RAN/Proc. ISP RAS, vol. 30, issue 6, 2018, pp. 259-274 (in Russian). DOI: 10.15514/ISPRAS-2018-30(6)-14

[16].Zilitinkevich S S, Hunt J C R., Grachev A A, Esau I N, et al. The influence of large convective eddies on the surface layer turbulence. // Quart. J. Roy. Meteorol. Soc., 2006. 132 1423- 1456.

[17].Huang J., Cassiani M., Albertson J. D. Analysis of coherent structures within the atmospheric boundary layer. // Boundary-Layer Meteorology. 2009, 131 147-171.

[18].Shah S., Bou-Zeid E. Very-Large-Scale Motions in the Atmospheric Boundary Layer Educed by Snapshot Proper Orthogonal Decomposition. Boundary-Layer Meteorology, vol. 153, issue 3, 2014 355-387.

[19].Yu H., Liu S.C., Dickinson R.E. Radiative effects of aerosols on the evolution of the atmospheric boundary layer. Journal of Geophysical Research, volume 107, issue D12, 2002, pp. AAC 3-1 - AAC 3-14.

20].Lauros J., Nilsson E.D., Dal Maso M., Kulmala M. Contribution of mixing in the ABL to new particle formation based on observations. Atmospheric Chemistry and Physics, vol. 7, 2007, pp. 4781-4792.

21]. Hancock P.E., Farr T.D. Wind-tunnel simulations of wind-turbine arrays on neutral and non-neutral winds. Journal of Physics: Conference Series, vol. 524. 2014, article no. 012166.

[22].Tellez-Alvarez J., Koshelev K., Strijhak S., Redondo J.M. Simulation of turbulence mixing in the atmosphere boundary layer and analysis of fractal dimension. Physica Scripta, vol. 94, no. 6, 2019, article no. 064004.

[23]. Hancock P.E., Pascheke F. Wind-Tunnel Simulation of the Wake of a Large Wind Turbine in a Stable Boundary Layer: Part 2, the Wake Flow. Boundary-Layer Meteorology, vol. 151, 2014, pp. 23-37.

[24]. Крючкова A.C., Стрижак C. B. Моделирование вихревого следа для случая двух модельных ветроустановок. Научный вестник МГТУ, том 21, № 1, 2018 г., стр. 40-48 / Kryuchkova A.S., Strijhak S.V. Modelling of turbulent wake for two wind turbines. Civil Aviation High Technologies, vol. 21, № 1, 2018, pp. 40-48 (in Russian.)
[25].Kryuchkova A., Tellez-Alvarez J., Strijhak S., Redondo J.M. Assessment of Turbulent Wake Behin Two Wind Turbines Using Multi-Fractal Analysis. Ivannikov ISPRAS Open Conference, 2017, pp. $110-116$.

[26].Strijhak S.V., Koshelev K.B., Kryuchkova A.S. Studying parameters of turbulent wakes for model wind turbines. AIP Conference Proceedings, vol. 2027, issue 1,_2018, article no. 030086

[27].Tellez-Alvarez J., Strijhak S., Kharchi R., Kryuchkova A., Redondo J.M. 3D Numerical Simulation of Wind Turbines and Fractal Dimension Analysis. In Proc. of the 2018 International Conference on Wind Energy and Applications in Algeria, 2018, $5 \mathrm{p}$.

[28].Strijhak S.V., Koshelev K.B., Kryuchkova A.S. Simulation of turbulent wakes in model wind farm with arbitrary location for wind turbines. Journal of Physics: Conference Series, vol. 1382, 2019, article no. 012043.

\section{Информация об авторах / Information about authors}

Константин Борисович КОШЕЛЕВ - кандидат физико-математических наук, доцент, старший научный сотрудник ИСП РАН с 2016 года. Сфера научных интересов: вычислительная гидродинамика.

Konstantin Borisovich KOSHELEV is candidate of physical and mathematical sciences, associate professor, senior researcher at the ISP RAS since 2016. Research interests: computational fluid dynamics.

Сергей Владимирович СТРИЖАК - кандидат технических наук, ведущий инженер ИСП РАН с 2009 года. Сфера научных интересов: вычислительная гидродинамика.

Sergei Vladimirovich STRIJHAK - candidate of technical sciences, leading engineer of the ISP RAS since 2009. Research interests: computational fluid dynamics. 